\title{
BIOLOGICAL BULLETIN
}

\author{
FURTHER EXPERIMENTS ON ADVENTITIOUS \\ REPRODUCTION AND POLARITY IN \\ HARENACTIS. \\ C. M. CHILD.
}

With Eleven Figures.

\section{INTRODUCTORY.}

In a paper which appeared in $1909^{1}$ I described the adventitious formation of new axes in the Californian actinian, Harenactis attenuata. These new axes have thus far been observed only under certain conditions, viz., in isolated pieces prepared in such manner that the oral cut surface of the body wall unites with the aboral about the whole circumference. In my earlier paper such pieces were designated as "rings."

During the past summer I again enjoyed the privileges of the laboratory at La Jolla and take this opportunity of expressing my thanks to the director, Professor Ritter, and to the trustees of the San Diego Marine Biological Association. During my stay at La Jolla I obtained results with "rings" from Harenactis which supplement my earlier work and extend and confirm my conclusions. These results form the subject of the present paper.

As described in my earlier paper, the rings are produced by isolating rather short, cylindrical pieces from the body of Harenactis by means of transverse cuts (Fig. I), and then removing more or less completely from these pieces the mesenteries and mesenterial muscles. The region of the body proximal to the esophagus is the most suitable for such operations, since here the mesenteries are well developed, bear large retractor muscles and extend into the enteron with free borders instead of being attached to the

${ }^{1} \mathrm{Ch}$ 1d, '09b, "Factors of Form Regulation in Harenactis attenuata, III., Regulation in 'Rings,'” Journ. Exp. Zool., VII 2, I909. 
esophagus, as in the more distal regions, or being only slightly developed, as in the more proximal, attenuated region.

The removal of the free borders brings about longitudinal contraction of the injured mesenteries and so approximates the oral and aboral cut surfaces of the body wall. This contraction
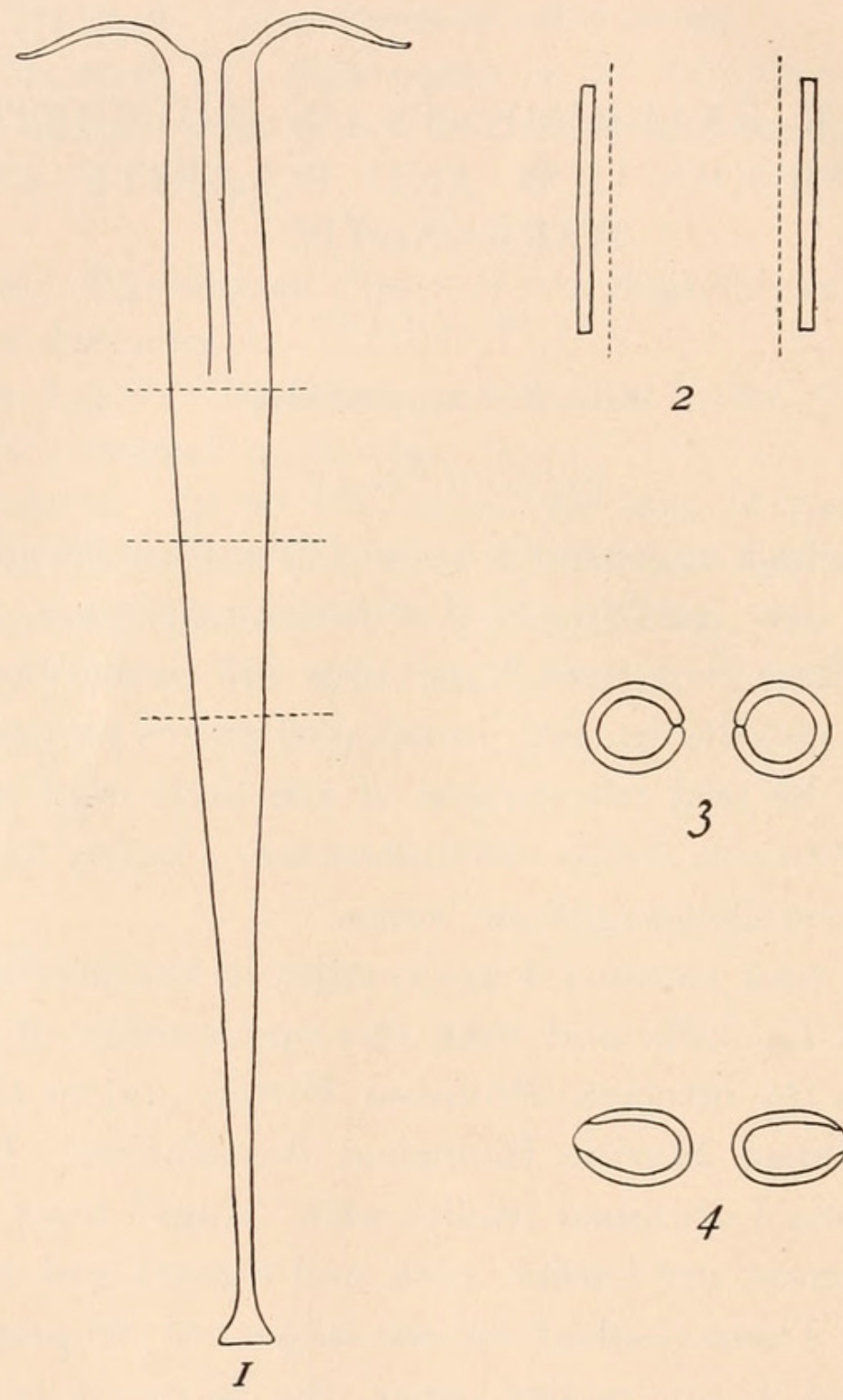

FIGS. I-4.

is merely a special case of the wound contraction so characteristic of the actinians, which I have described for Cerianthus and Harenactis. ${ }^{1}$ In my earlier experiments I removed merely such

${ }^{1}$ Child, '03, "Form Regulation in Cerianthus, I., The Typical Course of Regeneration," Biol. Bul.L., V., 5, I903.

'04, "Form Regulation in Cerianthus III., The Initiation of Regeneration," Biol. Bull., VI., 2, I904.

'08, "Form Regulation in Cerianthus asmari," Biol. Bull., XV., I, I908. 
portions of the mesenteries as protruded from the cut ends of the pieces, or accomplished the same result by other means. ${ }^{1}$ In the experiments of this summer greater care was taken to make certain that all the muscles were removed, and the free borders of the mesenteries - in most cases the greater part of the mesentery as well-were cut away. After these operations the pieces consisted of the body wall with only the bases of the mesenteries, $i$. e., those portions which lie nearest the line of attachment to the body wall. The nature of this operation is indicated approximately in the diagrammatic Fig. 2. This represents a typical longitudinal section of the body wall of the cylindrical piece: all the internal mesenterial structures between the two broken lines are removed, leaving only narrow strips of mesenterial tissue next to the body wall.

Such pieces form rings in almost every case, and nearly all of the rings give rise to tentacle groups. The result of the removal of the mesenteries is the approximation and contact of the oral and aboral cut ends of the body wall, as indicated in the optical section, Fig. 3. Union occurs between these cut surfaces and the body wall thus forms a closed ring with an opening through the center and with no connection between the enteron and the exterior. The enteric cavity becomes more or less distended with water after closure, undoubtedly in consequence of the passage of water through the body wall, and sooner or later a peculiar rotation of the parts about a circular axis situated in the enteric cavity of the ring occurs, as described in my earlier paper ('og b, pp. 356-357). The result of this rotation is a change in the position of the line of union between the oral and aboral ends from its original position around the central opening to the outer surface of the ring (cf. Figs. 3 and 4 of the present paper; in Fig. 4 the region of union between oral and aboral ends is indicated by the very thin body wall which is formed of new tissue). Very commonly the line of union, which is marked by the formation of more or less new tissue, finally comes to occupy a position somewhat upon the upper surface of the ring as this 'oga, "Factors of Form Regulation in Harenacis attemuata, I., Wound Reaction and Restitution in General and the Regional Factors in Oral Restitution," Journ. Exp. Zool., VI., 4, I909.

${ }^{1}$ Child, 'og $b$, Figs. $2-5$, Fig. 3I, also pp. 373-374. 
lies on the bottom of the dish (Fig. 4). If the ring is turned over, a new rotation often occurs, which brings the line of union into approximately the same relative position. As stated in my earlier paper ('ogb, p. 357), this appears to be an "attempt" at orientation with respect to the substratum, but since the original distal and proximal ends of the piece are united with each other and since, as is evident from the later processes in these pieces, the original polarity is decreased or almost eliminated by this union, any complete orientation is of course impossible.

Most of these rings give rise in the course of several weeks to groups of tentacles or single tentacles which lie along the line of union, but which may arise wholly on one side or the other, i.e., wholly from the original distal or the original proximal parts, or may involve both in the formation of a single tentacle group.

In some cases these groups of tentacles are without any close resemblance to the normal disc of Harenactis and they often show a more or less marked bilateral or biradial symmetry with respect to the line of union (Child, 'ogb, Fig. Io). More commonly, however, each group shows a more or less distinct radial symmetry, similar except as regards the number of radii, to the symmetry of the animal in nature (Child, 09b, Figs. I2, I5, I7-30). In these radially symmetrical tentacle groups mesenteries could in many cases be seen distinctly upon the small discs, their arrangement corresponding with the intervals between the tentacles. In some cases these were clearly continuations of the old mesenteries (Child, 'ogb, Figs. I8-20), but in other cases I was unable to find any such connection and was uncertain whether the formation of new mesenteries had occurred or not.

In my earlier experiments the largest number of tentacles in a radially symmetrical group was seven ('o9b, Fig. 29e) and in no case were mouth and esophagus present, though in one ring several groups showed a tentacle-like outgrowth in the center of the disc, where the mouth would normally appear. I suggested that this outgrowth might possibly represent an everted esophagus, but could not be certain as to its nature.

These tentacle groups were interpreted as representing new polarities, new body-axes, developing adventitiously in the region 
of union of the original distal and proximal ends of the piece, but failing to attain the usual form because of the peculiar conditions under which they arose.

In returning to these experiments this summer, I hoped to obtain tentacle groups which should approach more closely the usual form of Harenactis and thus leave no doubt that these outgrowths really represent new polarities, in short, that these phenomena in rings are really a form of asexual reproduction related to certain cases of the formation of adventitious structures in plants. These hopes were fully realized in one case, to be described below, and other tentacle groups among the large number obtained showed various points of interest.

\section{DESCRIPTION OF EXPERIMENTS.}

The case of greatest interest is one in which only one group of tentacles appeared upon the whole ring. This group was situated wholly upon one side - probably the oral - of the line of union, was radially symmetrical from the beginning and at first possessed seven or eight tentacles. During its growth, however, new tentacles appeared until the number reached sixteen. The tentacles were regularly arranged about a disc similar in appearance to that of the normal animal and in the center of the disc a mouth, opening into a short esophagus, appeared. On the disc sixteen mesenteries could be counted, most or perhaps all of these being new, as was evident both from their appearance and relation to other parts and from the impossibility that sixteen of the twenty-four old mesenteries should be involved in this new development from a small area localized on one side of the ring. Fig. 5 shows this new disc with the sixteen tentacles as it appeared twenty days after the operation. The line of union between the original oral and aboral ends of the piece is indicated by the dotted line. This new individual differs from the full grown animal in nature in the smaller number and unequal length of the tentacles and in the absence of a proximal end, but there can be no doubt that it represents a new body-axis, a new polarity.

After the formation of the disc it gradually became elevated above the surface of the ring by the growth of a cylindrical column beneath it. Fig. 6 is a diagrammatic optical section 
through the side of the ring on which the disc appeared; it shows the formation of the column beneath the disc. After twenty-five days the old tissues of the ring began to show signs of disintegration and the new individual was either poisoned or infected by the dead and putrefying tissue, and died. In no case thus far has it been possible to keep these outgrowths of the rings permanently alive, for the parts not involved in the new outgrowths always degenerate sooner or later and finally kill the new parts. In the case just described it was my intention to separate the new individual from the ring in order that it might if possible complete its development, but death occurred before this was done. If the opportunity of still further experiment arises, I

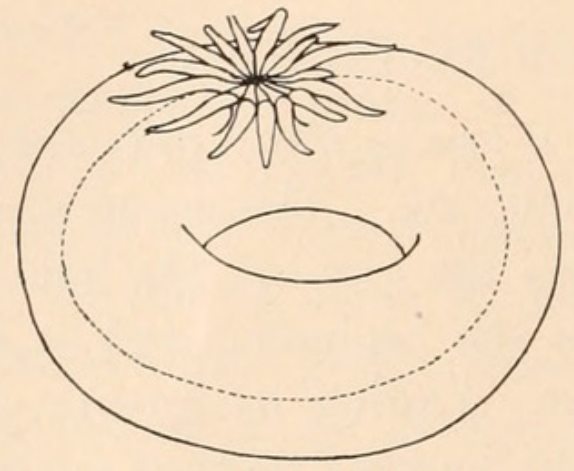

5

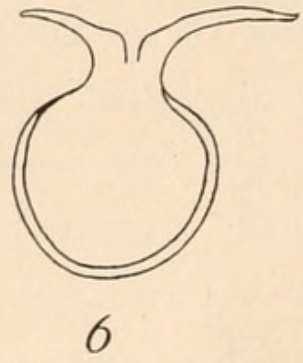

Figs. 5-6.

shall try the experiment of separating some of these adventitious buds, for they are such, from other parts of the ring. I have no doubt that under proper conditions they can be kept alive and fed so that development will proceed.

This case as it stands is, however, sufficient to demonstrate that new polarities may arise from these rings, from which the old polarity is in large measure eliminated. As was pointed out in my earlier paper, this is a form of reproduction which resembles more or less closely certain cases of the formation of adventitious structures in plants. Moreover, there can be no doubt that each new polarity arises in relation to local conditions in the growing tissues along the line of union between the original oral and aboral ends of the piece, though exactly what these conditions are, we do not at present know.

In the course of my experiments a large number of other adventitious tentacle groups was obtained, many of them with 
new mesenteries, but none with so large a number of tentacles as in the above case and none with mouth and esophagus. Besides the distinctly radially symmetrical groups, others, showing the most various degrees of radial and bilateral symmetry and asymmetry, appeared. Brief descriptions and figures of a few characteristic cases are added here.

Fig. 7 shows a case of considerable interest: here a disc with four radially arranged tentacles and with four well-developed mesenteries, but no mouth, appeared on one side of the line of union and was gradually elevated by the growth of a long column
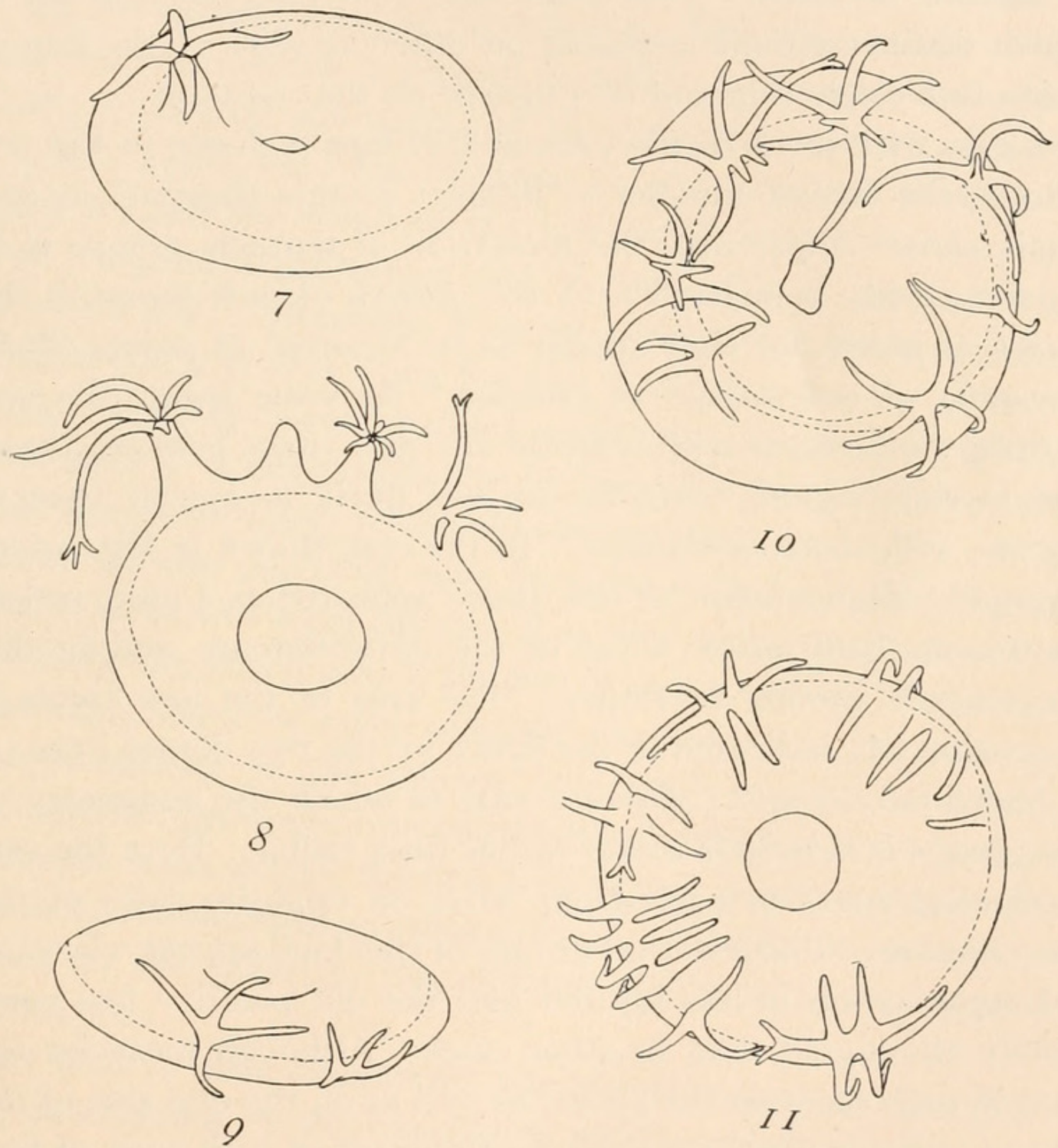

Figs. 7-II.

beneath it. No further tentacles were added during the life of this specimen and it finally died, apparently from the same causes as others. 
In Fig. 8, a ring with three tentacle-bearing outgrowths and one without tentacles is shown. The tentacles on two of the three outgrowths are radially symmetrical, though unequal in length and the discs show well-developed, radially arranged mesenteries, but are without mouths. The third group is less symmetrical and consists of only four tentacles. All the outgrowths appeared on one side, apparently the oral, of the line of union.

Fig. 9 shows a case, viewed obliquely from one side, in which two groups of four tentacles each appeared, one on either side of the line of union. This is the only case observed thus far in which distinct groups appeared on different sides of the line of union in a single ring and it is figured on that account.

A case with seven distinct tentacle groups is shown in Fig. Io. This is the largest number of distinct groups observed in any single case. Apparently the formation of tentacle groups is in general closely connected with the growth of new tissue in the region of union, for they appear to be localized at points where the areas of new tissue are greatest. At some points the two cut edges may unite with scarcely any new tissue between them, and in such regions tentacles are less likely to appear than in regions with more new tissue. In the case shown in Fig. Io an unusually large amount of new tissue appeared as a more or less continuous band about most of the circumference and in this the tentacle groups developed. The area of the new tissue is approximately indicated in the figure by the two dotted lines.

And finally, Fig. I I shows a case in which the symmetry of the groups is mostly bilateral rather than radial. Here the corresponding parts of each group arise on opposite sides of the line of union. I believe that groups of this kind appear when the old organization is less injured and the old polarity less completely eliminated than in other cases. Although tentacles appear in such cases on the aboral as well as on the oral side of the line of union, nevertheless the development of such rows of tentacles is manifestly a less extreme change from the "normal" arrangement with respect to the cut surface of the body wall than the formation of distinct, radially symmetrical groups on one side or the other of the line of union. It will be observed 
from the figure that in two cases the tentacles which apparently arise directly upon the line of union are forked at their tips: this means that they began their development as two separate tentacles, one on each side of the line of union, and later united to form a single tentacle. Similar conditions are shown in Fig. 8, $b$, and in Fig. Io of my earlier paper ('ogb).

\section{Conclusion.}

As regards the interpretation of these peculiar structures, there is little to add except by way of confirmation to the conclusions of my preceding paper on the subject. The outgrowths on the rings unquestionably represent a kind of reproduction which results from a certain degree of physiological isolation of parts, in other words from the decrease in physiological correlation, which is itself the result of the removal of important elements of the original organization and of the union of the oral with the aboral end. Moreover, it seems evident that the localization of the tentacle groups along the line of union is due to "chance" factors, probably in part to differences in the amount and rapidity of the growth of new tissues and on the other hand to differences in the degree of injury to the old organization.

That the new polarities have no direct relation to the original polarity is evident from the fact that they may arise from either the oral or the aboral side of the line of union, or from both sides. From these facts we must conclude that they are the result of local conditions in the regions concerned, and of conditions which are not primarily concerned with the original organization.

As regards symmetry, the new growth may be either bilaterally or radially symmetrical or asymmetrical. Clearly the symmetry, as well as the polarity, has nothing to do with the original organization.

And finally, these regulatory phenomena cannot be satisfactorily interpreted by the hypothesis which regards polarity and symmetry as essentially a summation of the polarities and symmetries of ultimate oriented particles or molecules. How, for example, shall we account on the basis of this hypothesis for the appearance of bilaterally symmetrical groups of tentacles in an organism which is naturally radially symmetrical. Further- 
more, how, unless we accept Driesch's entelechy, can we account for the complex changes in orientation of the particles which must occur in the development of a new polarity and radial symmetry from a small area of the oral or aboral end of the piece. If we turn to the process of crystallization for assistance, we meet difficulties, for we should expect, if the process of organic development is analogous to crystallization, that Harenactis material would in general conform to a particular type of arrangement, at least within certain limits of environmental change.

On the other hand, if we regard polarity and symmetry as molar phenomena resulting from the localization by any conditions or agents of certain metabolic processes differing in degree or kind, together with the physiological correlation resulting from such localization, we can readily understand how new polarities may arise without reference to the old, in response to local factors, and how bilateral symmetry may appear in one case and radial in another in the same organism.

The greater the extent to which we interfere with or destroy the old polarity and symmetry by removing or altering the localization of the original metabolic processes, the greater the possibility of the origin of new polarities and symmetries in response to local conditions.

In the recent experiments of Lillie, Morgan and others on the effects of centrifuging eggs, the polarity and symmetry of the eggs are, at least in certain cases, apparently not altered by the displacement of the visible granules. These results have led various experimental embryologists to adopt the hypothesis of orientation of molecules or particles as the basis of polarity and symmetry. As a matter of fact, however, the visible granules which are displaced in these experiments are merely the inactive or relatively inactive products of metabolism and so long as they are visible, have reference primarily to past activities. Their localization, in so far as they are localized with respect to an axis, suggests the more or less sharp localization along this axis of metabolic processes differing in degree or kind. There is no reason to believe that displacement of the granules by centrifuging alters essentially the localization of the processes, consequently the polarity and symmetry of the egg may remain 
unchanged, whatever the positions of the visible granules. As a matter of fact most, if not all eggs actually show a more or less sharp localization along the polar axis of processes differing in degree or kind: in general the region of the animal pole reacts more rapidly in various ways than the vegetative pole and between these two poles a gradation apparently exists. In every fragment of such eggs above a given size this gradation must exist in some degree, $i$. e., such fragments will retain the original polarity of the whole. If, however, we could control the size of isolated egg fragments and if it were possible to reduce the size of nucleated fragments indefinitely we should undoubtedly find a limit below which polarity would no longer be apparent. In every case where control of the size of isolated pieces is possible, such a limit has been found: unfortunately the egg cell is exceedingly unfavorable material for experiment along this line. It is at present extremely difficult and often impossible to isolate pieces of the egg of a certain desired size or from a certain desired region: moreover, if the isolated fragment is to live and show any developmental processes it must possess a nucleus. Manifestly the possibilities of investigating the polarity and symmetry of the egg by means of isolated fragments are very narrowly limited, as compared with the possibilities which simple organisms with elongated axes present, and we have no right to draw conclusions as to the nature of polarity from experimentation on eggs alone. Until we can actually prove that in eggs polarity does not decrease with decrease in size below a certain limit, or until we can demonstrate the orientation of particles or molecules by means of the polariscope, we have no adequate grounds for regarding polarity as anything but a molar phenomenon. Moreover, the fact already mentioned, that in all cases where control of the size of the isolated piece is possible, and where extended experimentation has been made, the phenomena of polarity do decrease with decreasing size, should suggest the necessity of caution in drawing conclusions from eggs alone, where both technical and natural obstacles to exact experimentation along these lines exist. Moreover, I fail to see how we can avoid accepting the evidence which the polariscope gives as possessing much greater weight than the results of experiment with our 
present crude technique, and this evidence is, except for certain highly specialized structures, $e . g$., the nerve fiber, negative, so far as I am aware. If an orientation of particles is the basis of organic polarity and symmetry, surely the polariscope must have given us more positive evidence of such orientation than we have yet obtained. In the absence of such evidence the orientationhypothesis possesses a metaphysical rather than a scientific character. We may juggle with the ultimate particles as we please and we may assume that they do all that is required to attain a certain result: unquestionably we shall succeed in interpreting all the phenomena of polarity in this way, but the value of our interpretations is chiefly academic, and scientific proof or disproof is impossible.

\section{Summary.}

I. The pieces of the actinian, Harenactis attenuata, which form "rings" by the union of oral and aboral ends about the whole circumference, after more or less complete removal of mesenteries and mesenterial muscles, may produce new discs with radially arranged new mesenteries and tentacles and mouth-opening and esophagus. Thus far the usual number of tentacles, twenty-four, has not been attained in any case, the largest number being sixteen. Such discs with from three to eight tentacles are of frequent occurrence, but the formation of mouth and esophagus has been observed only once. After the formation of the discs they may be gradually elevated from the surface of the rings by the development of a cylindrical column beneath them.

In addition to the well-developed discs, radially as well as bilaterally symmetrical and asymmetrical tentacle groups may arise along the line of union on either side, or the tissue of both sides may take part in the formation of a single group.

2. These further experiments with rings extend and confirm the earlier work. The new outgrowths on the rings represent a more or less close approach to new individuals and involve the establishment of new polarities and symmetries. They are to be regarded as a form of reproduction related to the formation of adventitious structures in plants. The localization of the outgrowths, as well as their polarity and symmetry, have no relation to the original polarity and symmetry, but are due to ocal conditions. 
The results of these experiments can be more readily interpreted in accordance with the hypothesis that polarity and symmetry are essentially molar localizations or gradation of processes along an axis or axes, than with that which regards polarity and symmetry as the effect of a summation of the individual polarities and symmetries of protoplasmic particles or molecules which are definitely oriented with respect to an axis or axes.

Hull Zoölogical Laboratory, University of ChicAGo,

November, I9I0. 


\section{$2 \mathrm{BHL}$ Biodiversity Heritage Library}

Child, Charles Manning. 1910. "FURTHER EXPERIMENTS ON ADVENTITIOUS REPRODUCTION AND POLARITY IN HARENACTIS." The Biological bulletin 20, 1-13. https://doi.org/10.2307/1535929.

View This Item Online: https://www.biodiversitylibrary.org/item/17126

DOI: https://doi.org/10.2307/1535929

Permalink: https://www.biodiversitylibrary.org/partpdf/19053

\section{Holding Institution}

MBLWHOI Library

\section{Sponsored by}

MBLWHOI Library

\section{Copyright \& Reuse}

Copyright Status: NOT_IN_COPYRIGHT

This document was created from content at the Biodiversity Heritage Library, the world's largest open access digital library for biodiversity literature and archives. Visit BHL at https://www.biodiversitylibrary.org. 